\title{
Modelling of the magnetic field structures and first measurements of heat fluxes for TEXTOR-DED operation
}

\author{
M.W. Jakubowski ${ }^{1,2}$, S.S. Abdullaev ${ }^{1}$, K.H. Finken ${ }^{1}$ and \\ the TEXTOR Team ${ }^{1}$ \\ ${ }^{1}$ Forschungszentrum Jülich, Institut für Plasmaphysik, Association EUROATOM-FZJ, \\ Trilateral Euregio Cluster, D-52428 Jülich, Germany \\ ${ }^{2}$ Also at: University of Opole, Institute of Physics, 45-052 Opole, Poland
}

Received 14 November 2003, accepted for publication 18 March 2004

Published 11 May 2004

Online at stacks.iop.org/NF/44/S1

DOI: $10.1088 / 0029-5515 / 44 / 6 / \mathrm{S} 01$

\begin{abstract}
The dynamic ergodic divertor (DED) was recently installed at the TEXTOR tokamak. One of the aims of the DED is to control and study heat and particle deposition on a plasma wall via modification of the plasma edge by external perturbation coils. Sixteen perturbation coils are mounted on the high-field side of the torus. The external magnetic perturbation creates a zone of chaotic field lines at the plasma edge by destroying several resonant surfaces. These structures have the properties of an open chaotic system while the field lines intersect the tokamak vessel. In order to study the topology of the field lines in different regimes, a set of tools called Atlas was created. Atlas uses a symplectic mapping technique to trace the magnetic field lines. The thermographic set-up is used to measure characteristic temperature patterns on the divertor target plates. The first measurements show the relation of the observed patterns with the results from the modelling with the Atlas codes.
\end{abstract}

PACS numbers: $52.55 . \mathrm{Fa}, 52.55 . \mathrm{Rk}$

\section{Introduction}

In a fusion reactor, the heat load density on the plasma facing components is considered a challenging problem [1]. The high heat load results from the narrowness of the characteristic decay length of the power, which is for all tokamaks of the order of $1 \mathrm{~cm}$. The wetted area of the divertor can be enhanced somewhat by flux expansion and by inclination of the target tiles. It would be beneficial to enhance the width further, e.g. by 'deconfining' the edge of the plasma (i.e. by degrading the magnetic confinement in the outer volume of the tokamak). This idea led to an alternative concept of the ergodic divertor in which the external coils create an additional magnetic perturbation field. Until now only a few fusion machines [2], such as TEXT [3], Tore Supra [4], JFT-2M [5] and Hybtok [6], have investigated this type of divertor.

A new experimental device has recently been installed at the TEXTOR tokamak - the dynamic ergodic divertor (DED) [7]. Sixteen perturbation coils (plus two compensation coils) are installed on the high-field side (HFS) of the machine. As a unique feature, the DED allows for a rotation of the perturbation field with different frequencies; it is very flexible in choosing the mode structure of the perturbation field. The resonant $m / n$ perturbation field (with $m \in\langle 10, \ldots, 14\rangle$, $n=4$ ) was chosen during the initial operation. The amplitude of the perturbation field for this mode decreases with the distance from the coils as $\delta B_{\mathrm{r}} \propto\left(r / r_{\text {coil }}\right)^{m_{\text {eff }}-1}$, where $m_{\text {eff }} \approx$ 20. Because of the high multipolarity, the field decays within a few centimetres. Much deeper penetration of the perturbation field can be obtained by the $3 / 1$ or $6 / 2$ modes, which are also possible. Without the perturbation field, the magnetic field lines of a tokamak form a set of shell-like magnetic surfaces. When the axis-symmetry is broken, e.g. by a perturbation field, the field lines no longer stick to 'their' surface, but fill a volume. This creates a volume in the plasma edge where the field lines have stochastic properties, which significantly changes the plasma properties in the edge.

At the outermost boundary, where the near-field effects are significant, magnetic field lines have short wall-to-wall connection lengths. In chaos theory, a characteristic length for the separation of neighbouring orbits is the Kolmogorov length. If the intersection of the field lines with the walls is shorter then the Kolmogorov length, the transport of energy and particles is not of diffusive character, as in the ergodic zone, but has rather a convective/conductive character. In contrast to the proper ergodic zone, the connection lengths of the magnetic 
field lines have smooth and continuous properties with some sharp boundaries [8,9]. The structure of the different magnetic regions created by the DED is presented in figure 1 .

The goal of the DED in static and quasi-static operation is the redistribution of the heat fluxes to larger areas and the avoidance of avoid peak heat loads. The deposition patterns are driven by the structure of the laminar zone; therefore, it is necessary to have good knowledge of the topology of the laminar region.

To achieve a good understanding of the physics of the DED we have analysed the structure of the magnetic field. Several numerical [7] and analytical [10] attempts have been performed. Monte Carlo codes were developed in order to calculate the plasma properties (E3D [11,15] and EM3D [16] codes), as well as a finite element method [12]. The numerical methods, which are based on a field line tracing technique, reveal detailed structures of the ergodic and laminar regions. However, the field line tracing codes need much CPU time to perform calculations for studying the structures of the ergodic or laminar zone of the plasma. In order to shorten the required time for calculating the structures of the magnetic field in the plasma edge, a mapping scheme was developed [10]. This

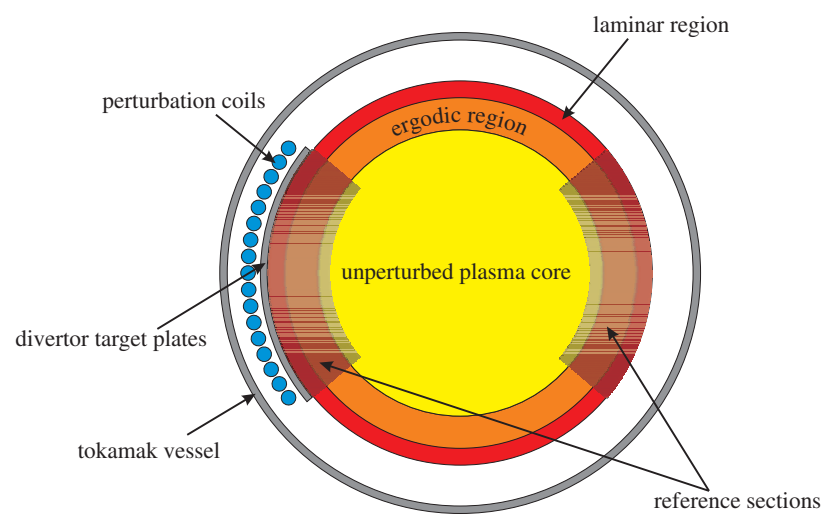

Figure 1. The sketch of the different regions created by the superposition of the tokamak equilibrium and DED perturbation field presented in a poloidal cross-section. method is derived from the continuous Hamiltonian equations and it is based on a canonical transformation of variables and on perturbation theory. The mapping allows for a visualization of the varied structures generated by the DED. Atlas is our means for this representation; it shows both the ergodic and laminar zones as well as the magnetic footprints.

Atlas consists of codes for evaluating the structures of magnetic fields induced by the DED in the outermost regions of the TEXTOR plasma. For visualizing the ergodic region, Poincaré sections were used, which are native representations for mapping techniques [13]. Poincaré plots are not suitable for studying the regions with short connection lengths of the field lines. In order to analyse the flux tubes at the very edge of the plasma, so-called laminar plots were developed: a field line is traced from the initial conditions until it hits the wall (in both directions), and the calculated connection length is marked on a graph at a position defined by the initial conditions. These images are important for understanding the heat and particle deposition patterns. For representing the transport properties in the plasma edge, a field line tracing program was developed which allows us to identify different flux tubes in the laminar zone and in the footprints structure.

In order to validate the calculations of the Atlas software a thermographic measurement system was developed. The main part of the set-up is a fast infrared camera equipped with an InSb focal plane array. The characteristic structure of the footprints stripes has been known since the very first experiments with the DED.

\section{The experimental set-up}

The DED (as shown in figure 2) consists of four quadruples of coils mounted on the high field side (HFS) inside the torus. Each coil is wound helically once around the torus following the field lines on the $q=3$ surface. Every coil is supplied individually from the outside of the vessel with a 4-phase current, with an amplitude of up to $15 \mathrm{kA}$. For the dc 12/4 mode, two current distributions are possible (see table 1), both approximate sinusoidal current distributions. The current

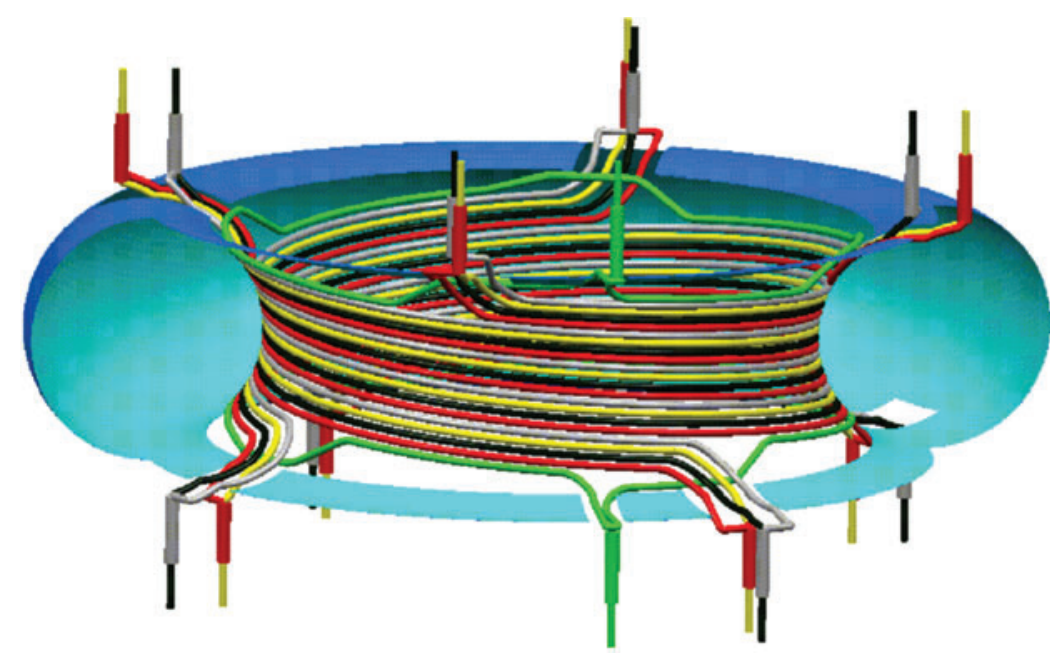

Figure 2. Sketch of the DED coils. The DED consists of 16 coils; the four different colours indicate the four phases of the electrical currents $\left(0^{\circ}, 90^{\circ}, 180^{\circ}, 270^{\circ}\right)$, which are supplied to the DED coils. For the correct vertical plasma positioning, two compensation coils (green) are added. 
Table 1. The current distribution creating $12 / 4$ mode of the perturbation field for 8 of 16 coils. Presented current distributions impose sinusoidal perturbation field to the plasma.

\begin{tabular}{lllllllllll}
\hline No & Coil & 1 & 2 & 3 & 4 & 5 & 6 & 7 & 8 & $\cdots$ \\
\hline 1 & $I_{\text {DED }}[\mathrm{kA}]$ & +15 & 0 & -15 & 0 & +15 & 0 & -15 & 0 & $\ldots$ \\
2 & $I_{\text {DED }}[\mathrm{kA}]$ & +15 & -15 & -15 & +15 & +15 & -15 & -15 & +15 & $\cdots$ \\
\hline
\end{tabular}

distribution (1) represents the current at one phase of the ac current; the distribution (2) is only possible for the dc case and has twice the amplitude of (1).

In the modelling, the following equation for current distribution was used:

$$
I_{j}=I_{\text {DED }} \sin \left(\frac{\pi j}{2}\right), \quad(j=1, \ldots, 16),
$$

which assumes a symmetric distribution of the coil feeders

The main perturbation modes (with poloidal mode numbers $m=10, \ldots, 14$ and toroidal mode number $n=4$ ) are centred at the respective magnetic flux surface with $q=12 / 4$. The base mode $12 / 4$ was chosen as a standard operational scenario due to technical constraints (such as current density, skin effect and heat capacity) and physical requirements. One of the most important physical requirements is not to disturb a $q=2$ surface (this could lead to interactions with intrinsic MHD modes and affects the plasma stability). The 4-phase current can be operated at several frequencies: $\mathrm{dc}, 50 \mathrm{~Hz}$ and at seven frequencies in the band $1-10 \mathrm{kHz}$. Because the feeders are bundled to 4 input and 4 output parts, two compensation coils are added for the plasma equilibrium. All coils are covered by the graphite tiles which form the divertor target plates. The radius of the coils is $r_{\text {coils }}=53.25 \mathrm{~cm}$, and the radius of the tiles is $r_{\mathrm{DED}}=47.7 \mathrm{~cm}$. A detailed report about this device is given in [7].

\section{Mapping scheme}

In this section we discuss a mapping method which was used to construct the Atlas software. Detailed discussions of the method are presented in $[10,11]$.

The 'propagation' of the field line is written as

$$
\frac{\mathrm{d} \bar{x}}{\mathrm{~d} s}=\frac{\vec{B}(\vec{x})}{|\vec{B}(\vec{x})|},
$$

where $\vec{x}=(r(s), \theta(s), \varphi(s))$ and $s$ is the length along the field line. Field line equations can be formulated in Hamiltonian form by introducing a new coordinate system with so-called intrinsic coordinates $\left(\psi, \theta^{*}, \varphi\right): \theta^{*}$ is intrinsic poloidal angle, $2 \pi \psi$ is the toroidal magnetic flux enclosed by the flux surface $\psi$, and $\varphi$ is a toroidal angle. In the intrinsic coordinates, the pitch of the field lines at one magnetic flux surface is constant. The spatial coordinates of the field lines are unique functions of new coordinates $\vec{x}=\vec{x}\left(\psi, \theta^{*}, \varphi\right)$. Using the intrinsic coordinates one can write a divergence-free magnetic field in a canonical form: $\vec{B}=\nabla \psi \times \nabla \theta^{*}+\nabla \varphi \times \nabla H$, where the $H$ is a Hamiltonian of the field line equations

$$
\frac{\mathrm{d} \theta^{*}}{\mathrm{~d} \varphi}=\frac{\partial H}{\partial \psi}, \quad \frac{\mathrm{d} \psi}{\mathrm{d} \varphi}=\frac{\partial H}{\partial \theta^{*}} .
$$

The Hamiltonian consists of two parts and can be written in the following way:

$$
H\left(\psi, \theta^{*}, \varphi\right)=H_{0}(\psi)+\varepsilon H_{1}\left(\psi, \theta^{*}, \varphi\right),
$$

in which $H_{0}$ is a part describing the equilibrium tokamak field and $\varepsilon H_{1}$ represents the perturbed part of the Hamiltonian ( $\varepsilon$ is a small, dimensionless parameter) [13]. The tokamak equilibrium field is axis-symmetric and depends only on the toroidal flux $\psi$. Therefore $H_{0}$ is determined only by the safety factor

$$
q(\psi)-H_{0}=\int q^{-1} \mathrm{~d} \psi
$$

The perturbation part $\varepsilon H_{1}$ can be presented as a Fourier series:

$$
H_{1}\left(\psi, \theta^{*}, \varphi\right)=\sum_{m, n} h_{m n}(\psi) \cos \left(m \theta^{*}-n \varphi\right),
$$

where $h_{m n}$ is the spectrum of the perturbation. The safety factor values and the spectrum of the perturbation are calculated by integrating the field lines for one poloidal turn, using the Runge-Kutta integration scheme. The integration is performed for a number of flux surfaces lying in the plasma outer region. The intermediate values are calculated using the cubic-spline method.

To study the trajectories of the field lines a symplectic mapping method $[10,11]$ was used, which is very convenient for studying the Hamiltonian systems [13]. A properly constructed map scheme is flux preserving and much faster than traditional integration methods.

\section{Properties of the laminar and ergodic regions}

\subsection{Poincaré and laminar plots}

The traditional technique to visualize the outcome of the mapping procedure is the so-called Poincaré plot, which reduces the problem by one dimension. The plot is created by tracing one or more field lines and marking the intersections of the field line(s) with a pre-selected poloidal section. Figure 3 illustrates the method of creation of the Poincaré plot and an example in polar coordinates. In figure $3(a)$ the tokamak torus is presented with four poloidal sections, the distance between them being $90^{\circ}$. Because of the toroidal symmetry of the perturbation field $n=4$ one can use all those planes to mark the intersections of the field lines. This procedure reduces the computation time by a factor of 4 . The field line is traced either to a maximal number of steps (e.g. 800 steps) or until it intersects the divertor target plates at $r_{\mathrm{div}}=47.7 \mathrm{~cm}$. This latter method 'simulates' the effect of neutralization of a particle hitting the wall. An example obtained by this procedure is shown in figure $3(b)$. For a better understanding of the plot geometry, the DED coils, the tokamak vessel and the temperature profile (obtained from the EM3D code) are 


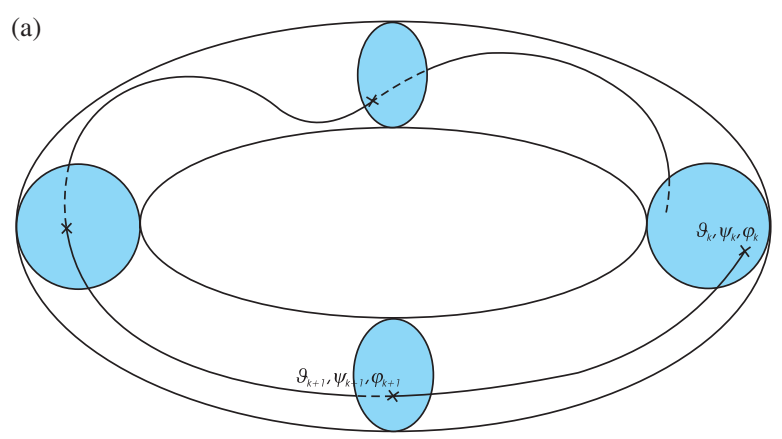

(b)

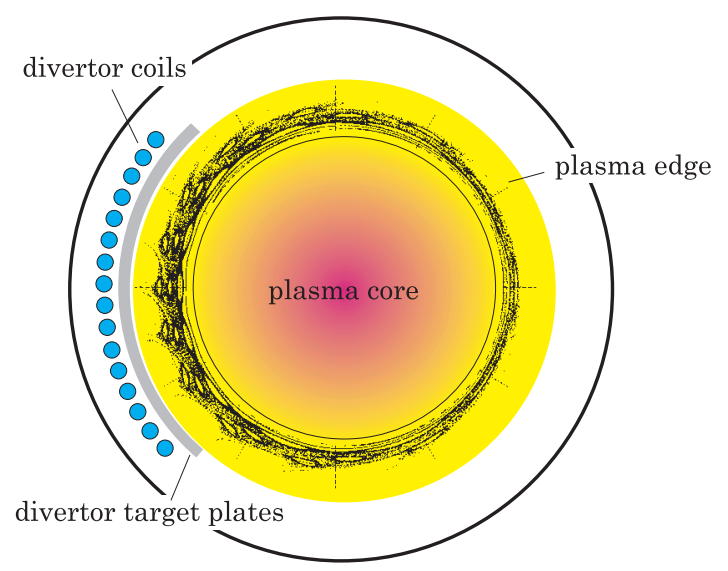

Figure 3. The Poincaré plot of the magnetic field lines presents a topology of the magnetic field: $(a)$ illustration of the method, because of the four-fold symmetry for $12 / 4$ mode, the period for graph is $\pi / 4 ;(b)$ example of the Poincare plot for full perturbation current superimposed onto the sketch of the poloidal section of TEXTOR vessel and the DED coils.

superimposed. One can see that the radial extension of the perturbed volume is small compared to the plasma diameter; therefore, it is convenient to cut and unfold the picture and study only the plasma boundary. The result is shown in figure 4 . In this figure the abscissa is the poloidal angle from $0^{\circ}$ to $360^{\circ}$ and the ordinate is the minor radius. In figure 4 the DED is marked as a rectangle on the top of the graph. The case presented here is for $I_{\mathrm{DED}}=15 \mathrm{kA}$ and of the equilibrium field of plasma for $I_{\mathrm{p}}=450 \mathrm{kA}, B_{\varphi}=1.88 \mathrm{~T}, \beta_{\mathrm{pol}}=0.0$. The visible island chains are marked on the right-hand side ordinate with the corresponding value of the safety-factor; e.g. the $m / n=10 / 4$ mode contains 10 islands. Each island chain is generated by different modes. The number of modes is related to the $q$-values of the resonant surface. The island chain created on the $q=12 / 4$ surface is completely destroyed.

The region of the perturbed field lines can be divided into two different zones. The volume $(42 \mathrm{~cm} \leqslant r \leqslant$ $46 \mathrm{~cm}$ ), where the field lines have very long connection lengths (roughly, more than 5 poloidal turns), is called ergodic. In this figure all characteristic features of the ergodized edge of the TEXTOR-DED can be seen: the typical picture of the ergodic zone is the volume with mixture areas with completely destroyed flux surfaces and some remnants of the islands. The trajectories of the field lines in the ergodic region are irregular. Initially neighbouring field lines will deviate from each other substantially and in an unpredictable way. This is in contrast to field lines in the good confinement zone and in islands. There, the field line trajectories remain inside the island chain and are separated from the stochastic areas. They still maintain a regularity. Because a limiting wall (divertor target plates) is placed inside the ergodic zone, the character of the field lines is changed to an 'open chaotic system'. Even though the field lines may remain for very long paths inside the ergodic zone, they will finally hit the wall. It was found that the field lines leave the ergodic zone in a well-ordered way, namely along so-called fingers (see figure 4). They are rather thin and surround the laminar zone.

In the region closest to the DED coils $(r \geqslant 46 \mathrm{~cm})$, the near-field effects play a dominant role. The field lines have very short wall-to-wall connection lengths. In the method that was used, the field line tracing stops after the intersection with the wall. Therefore the laminar zone appears as a region empty of Poincaré points (see, e.g. figures 3 and 4).

To understand the properties of the laminar regions a new technique (consequently named a laminar plot) was developed. The imaging is based on counting the connection lengths for each of the traced field lines. The representation is similar to that of the Poincaré plot. Three regions are of interest and are marked on figure 1 as the reference sections. These are:

- the footprints of the intersections of the magnetic field lines with the divertor target plates,

- the plane containing the stagnation points for the particle flow on the low-field side (LFS) for field lines with connection lengths of odd poloidal turns,

- the plane containing the stagnation points for the particle flow on the HFS for field lines with connection lengths of even poloidal turns.

A grid is created on those sections and each knot of the grid defines the initial coordinates for the tracing procedure. The typical grid contains $200 \times 200$ points; therefore, the distance between adjacent points is less than $2^{\circ}$ in the poloidal direction and about $2.5 \times 10^{-2} \mathrm{~cm}$ in the radial direction. The field line is traced in both directions until it intersects the wall. A connection length is assigned to the knot. After all knots have been assigned numbers a contour plot is created-an example is shown in figure 5. The abscissa and the ordinate represent the same variables as in figure 4 . Flux tubes with different connection lengths are marked with different colours. One can recognize on this plot typical structures of the laminar zone. The ergodic region from the Poincaré plot corresponds to the dark red region in the laminar plot. The fingers appear as very thin structures surrounded by the flux tubes belonging to the laminar zone (green and blue areas). The size of the flux tubes is decreasing with increasing connection length of the field lines forming the flux tube. The laminar regions exist mainly in the outer region; however, some of the flux tubes can penetrate into the ergodic region. The field lines marked in dark blue leave the plasma region very soon; they form a private flux region. The properties of the private flux zone are expected to be similar to those of a poloidal divertor. The case shown in figure 5 was calculated for similar conditions as the Poincaré plot in figure 4.

\subsection{Properties of the perturbed volume}

In figure $3(b)$ it is observed that the width of the perturbed volume is small compared to the plasma minor radius, usually 


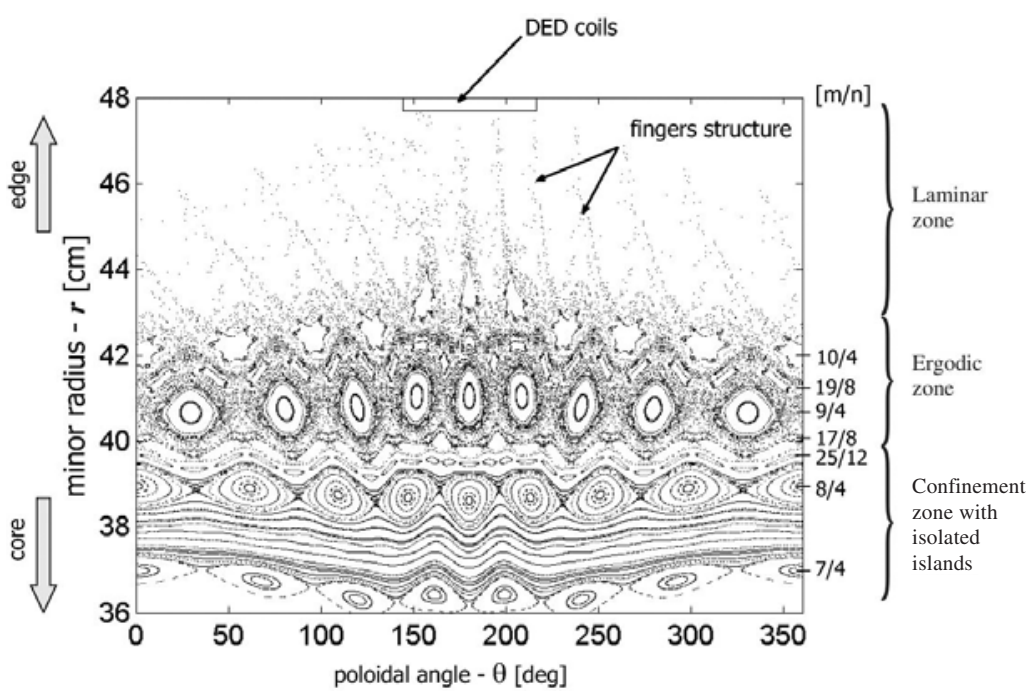

Figure 4. Poincaré plot for the magnetic field lines in the plasma edge.

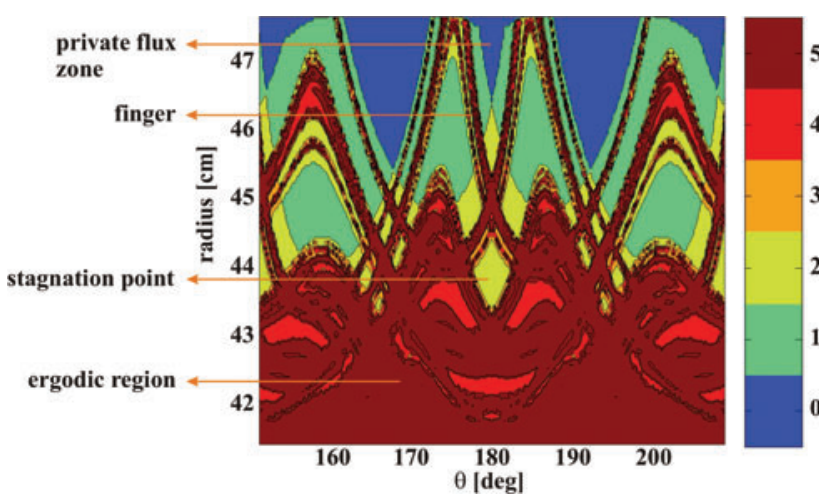

Figure 5. The laminar plot for the field lines in the edge of the TEXTOR plasma on the HFS. Different colours mark different connection lengths.

only a few centimetres. The size of the 'deconfined' boundary strongly depends on the DED currents amplitude and plasma parameters. The topology generated by the TEXTOR-DED is mainly determined by:

- the position of the resonant flux surfaces, which is defined by the interplay of the plasma current $I_{\mathrm{p}}$ and the toroidal field $B_{\varphi}$ (see figure 6);

- the pitch angle of the field lines and the differential Shafranov shift, which define $\beta_{\text {pol }}$; thus the spectrum of the perturbation strongly depends on a value of $\beta_{\mathrm{pol}}$. This is illustrated in figure 7.

In figure 6 the dependence of the radial positions on either the plasma current (with fixed $B_{\varphi}=2.2 \mathrm{~T}$ ) or the strength of the toroidal field (with fixed $I_{\mathrm{p}}=440 \mathrm{kA}$ ) are presented. The values of the perturbation spectrum on the resonant $q=3$ surface for three different values of $\beta_{\mathrm{pol}}$ are shown in figure 7 .

In the ergodic region, where the flux surfaces do not exist any more, the transport can be described by the field line diffusion coefficient $D_{\mathrm{FL}}$ [11]. The perpendicular transport is strongly enhanced there. The connection lengths of the magnetic field lines can be very long and reach hundreds of poloidal turns. The structure of the connection length

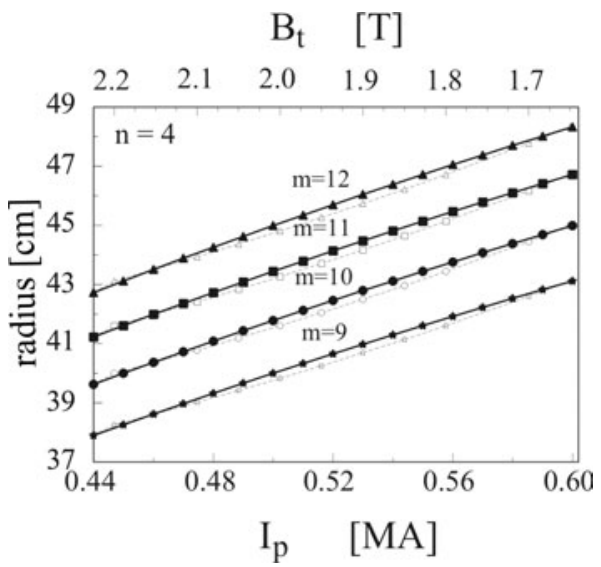

Figure 6. The position of the resonant surfaces as a function of: $(-)$ plasma current with fixed $\left.B_{\varphi}=2.25 \mathrm{~T} ;(--)^{-}\right)$toroidal field with fixed $I_{\mathrm{p}}=440 \mathrm{kA}$.

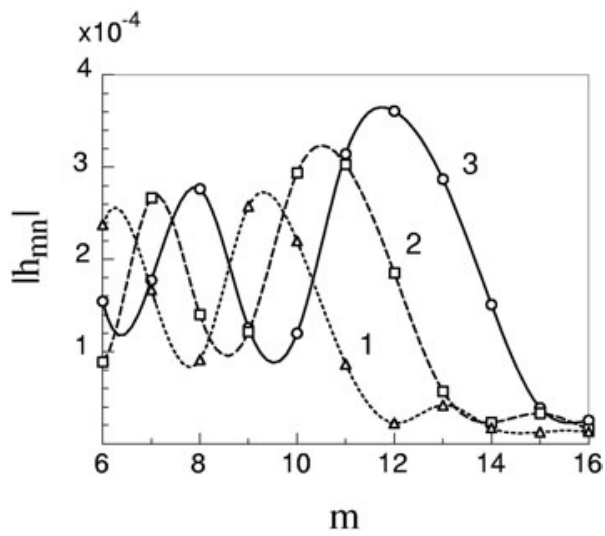

Figure 7. Spectrum of perturbation for three different values of beta poloidal: $(\longrightarrow) \beta_{\mathrm{pol}}=0.2,(--)_{\mathrm{pol}}=0.6,(\cdots \cdots) \beta_{\mathrm{pol}}=1.0$.

is fractal; i.e. neighbouring points can have very different connection lengths. This is different from the laminar zone. There are relatively large areas in which the field lines have the same connection lengths. The largest areas in which the 

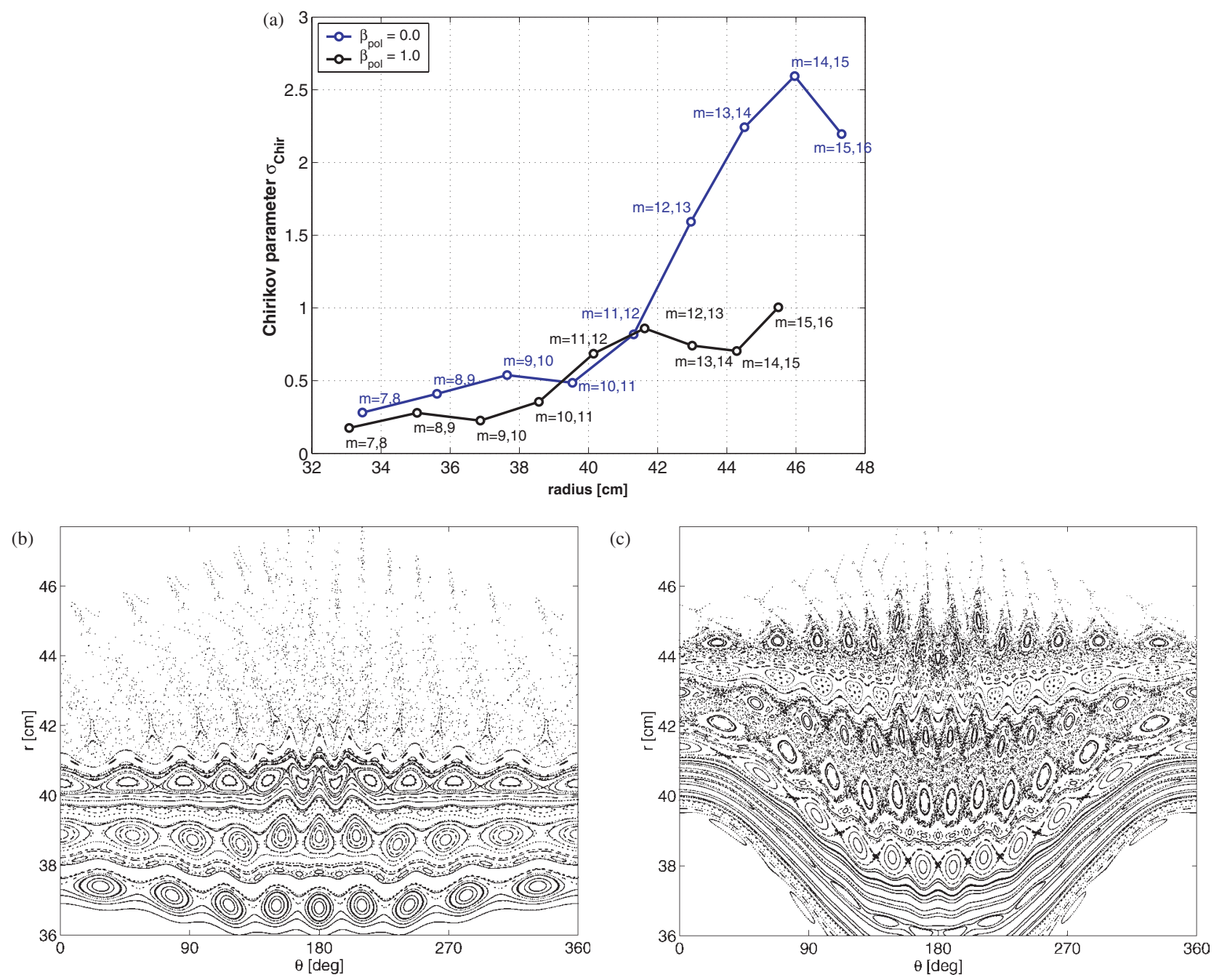

Figure 8. (a) The Chirikov parameter versus the plasma radius for two values of poloidal beta. Labels number interacting island chains $(n=4)$. $(b)$ and $(c)$ Poincaré plots for the same conditions as the curves shown in $(a)$.

field lines have the same connection lengths are generally characterized by the shortest connection lengths. There exist, however, boundaries where one jumps from an area of, e.g. a single turn connection length to one of double turn connection lengths. The individual flux tubes are quite similar to a normal scrape-off layer. However, at the boundaries large gradients in the flow velocity may occur.

A quantitative measure of ergodicity is given by a Chirikov parameter - a ratio of the widths of islands from two neighbouring chains and the distance between the chains [14]

$$
\sigma_{\text {Chir }}=\frac{(1 / 2)\left|\Delta \psi_{m+1, n}+\Delta \psi_{m, n}\right|}{\left|\psi_{m+1, n}-\psi_{m, n}\right|} .
$$

The Chirikov parameter is a simple measure of the ergodicity. For the case $\sigma_{\text {Chir }}<1$ the islands do not overlap and there is no ergodization. If $\sigma_{\text {Chir }} \geqslant 1$, the islands overlap and the flux surfaces between the chains are destroyed; the stochastic domain is established. In figure $8(a)$ the Chirikov parameter is plotted against the plasma radius for two different cases. Both calculations were made for $I_{\mathrm{p}}=360 \mathrm{kA}, B_{\varphi}=1.88 \mathrm{~T}$, $R_{0}=1.74 \mathrm{~m}, I_{\mathrm{DED}}=15 \mathrm{kA}$, but for different values of beta poloidal. The level of ergodization in case of $\beta_{\mathrm{pol}}=0.0$ is much higher than for $\beta_{\mathrm{pol}}=1.0$. As was shown in figure 7, the amplitudes of the perturbation spectrum are higher for lower $\beta_{\mathrm{pol}}$. One reason for this is that the pitch angle of field lines depends on the value of $\beta_{\mathrm{pol}}$ (for $\beta_{\mathrm{pol}}=0.0$ field lines at $q \approx 3$ are parallel to the DED coils). Also the Shafranov shift, which defines distance between flux surfaces, is a function of $\beta_{\mathrm{pol}}$. For higher $\beta_{\mathrm{pol}}$, the Shafranov shift is larger and the resonant surfaces are shifted away from the perturbing DED currents. Poincare plots of the magnetic field for these two cases are shown in figures $8(b)$ and $(c)$. In the case of $\beta_{\text {pol }}=0.0$, shown in figure $8(b)$, the laminar layer dominates. In the case of $\beta_{\mathrm{pol}}=1.0$, shown in figure 8(c), the effect of the DED current is drastically reduced and ergodic regions around main resonances are established.

The width of the perturbed volume is a nonlinear function of the plasma current and the poloidal beta. This relation is presented in figure 9 . The calculations were made with $B_{\varphi}=1.88 \mathrm{~T}, R_{0}=1.74 \mathrm{~m}$. The maximum width of the perturbed layer is about $7-8 \mathrm{~cm}$ and for higher beta it is reached with higher plasma currents. It can be also explained by the influence of $\beta_{\text {pol }}$ on the pitch angle and Shafranov shift. The characteristic jumps in the width at increasing plasma current 


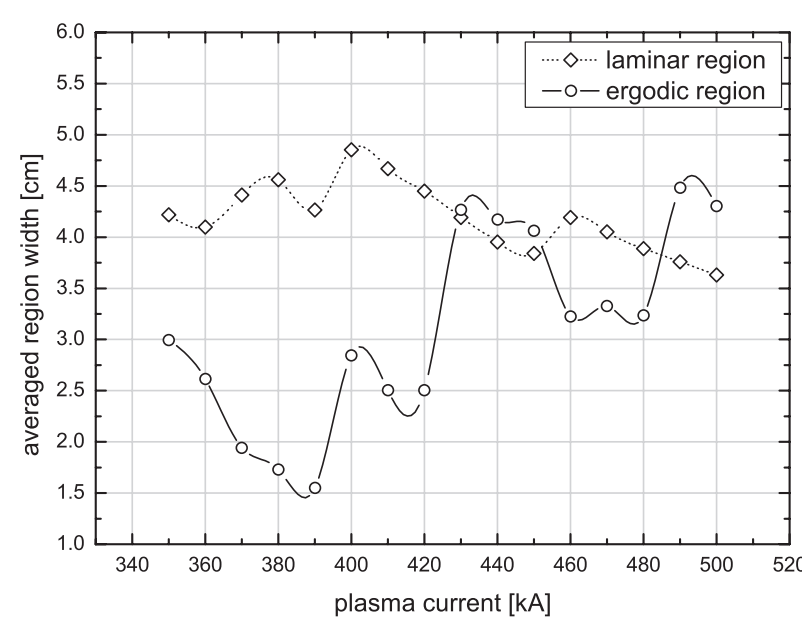

Figure 9. The width of the perturbed volume versus the plasma current.

are connected with 'loss' of the resonant surfaces from the plasma bulk. The local maxima are always reached when the resonant surface is exactly at the plasma edge.

A question that we need to address is about the relationship between the laminar and ergodic regions. As the transport properties of the two regions are dissimilar, we study cases with extended laminar zone and with dominating ergodic zone. In the first case the action of the divertor should be similar to that of the poloidal divertor, while in the latter case chaotic field lines contribute significantly to the perpendicular heat and particle transport coefficients. The plasma current, which defines the position of the resonant surfaces, serves as a parameter to establish diverse scenarios.

The dependence of the averaged width of the laminar and ergodic volumes is plotted against the plasma current in figure 10. The calculations are held for the same conditions as in figure 9. The width of the laminar zone is defined as the deepest penetration of the flux tubes with connection lengths of one or two poloidal turns, depending on which flux tube penetrates more deeply. The reason for considering only the flux tubes with connection lengths of at most two poloidal turns is that they are big enough to contribute to the scrapeoff layer. Their size is larger than the characteristic diffusion length. The typical diffusion length corresponds to the width of the scrape-off layer and amounts to about $1 \mathrm{~cm}$. Using the definition given here, the width of the laminar zone shown in figure 5 would be about $4 \mathrm{~cm}$, while the two-poloidal-turn flux tubes penetrate the plasma up to $r \approx 43.5 \mathrm{~cm}$. As one can see in figure 10 the ergodic regime controls the plasma edge at lower plasma currents, and as the plasma current increases the laminar regime becomes dominant. The example of a laminar plot for the dominant ergodic zone is shown in figure 11(a). The case with a dominant laminar region is illustrated in figures $11(b)$ and $(c)$. If the proper laminar zone is developed, one expects that the heat and particles originating in the plasma centre first leave the core through the ergodic region and then reach fingers (see figure 5). Because of its thin structure they diffuse to areas with short connection lengths-one or two poloidal turns - and they are guided parallel to the field lines to the wall.

As already mentioned above, the area with stagnation points of an odd number of poloidal turns is in the outer

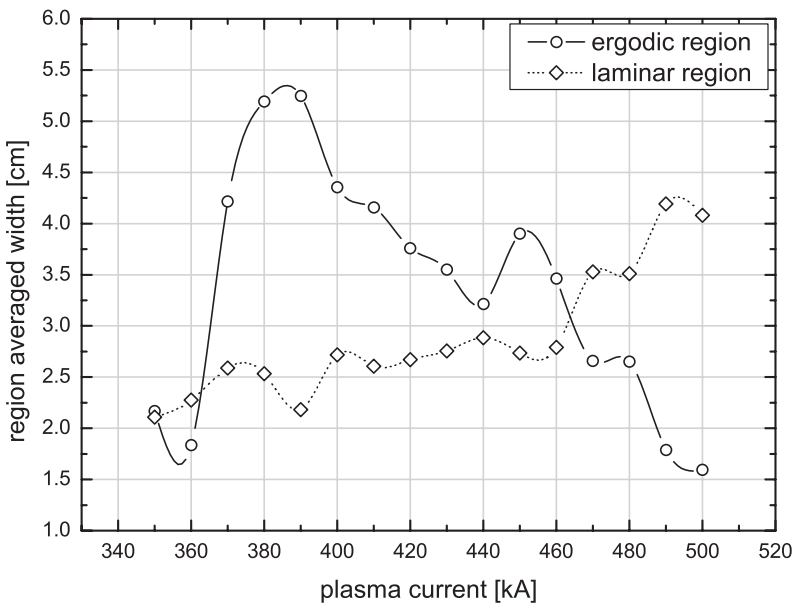

Figure 10. Averaged region width versus the plasma current for ergodic and laminar layer for $\beta_{\mathrm{pol}}=1.0$.

midplane, while those of an even number are in the inner midplane. In figures $11(b)$ and $(c)$ the stagnation areas can be identified. The areas which belong to the same flux tube are marked with the same number. As can be seen in figure 11(b), the stagnation area for the 1 poloidal turn region is at $\theta=0^{\circ}$ and $r=44 \mathrm{~cm}$. The laminar plot shown in figure $11(\mathrm{c})$ is shifted by $45^{\circ}$ toroidally. The stagnation area of 2 poloidal turn appears at $\theta=0^{\circ}$ and $r=43.5 \mathrm{~cm}$.

A question is how the topology of the magnetic field in the plasma boundary will be reflected in the heat and particle patterns. To study that problem, a similar technique was used as for the laminar zone. The contour plot of field lines' connection lengths is plotted in the target plates' plane $(\varphi, \theta)$. The revealed structure shows five narrow, helical stripes parallel to the DED coils. The field lines coming from the plasma hit the wall along these stripes. Most of the divertor surface is hit by the field lines, which were released from the same surface. It forms a zone which is analogous to the private flux zone of the standard SOL. Examples shown in figure 12 are calculated for the same conditions as for the laminar plots in figure 11. The abscissa represents toroidal angle and the ordinate poloidal angle. Only $\frac{1}{4}$ of the full toroidal angle is plotted because of the four-fold symmetry. The structure of power stripes strongly depends on the level of ergodization. For the case with low ergodization the stripes are thinner, whereas, if the level of ergodization is increased then they start to become wider and at some point they split into two parts. This is shown in figure 12(b). Field lines which have initial conditions at one part hit the wall along the second part. It is another hint to treat the laminar region as similar to a scrapeoff layer of a conventional divertor. Calculations performed by different numerical codes $[12,15,16]$ suggest that power and particle influxes follow the one- or two-poloidal-turns flux tubes and form characteristic patterns which are similar to the footprints structure.

\section{First experimental results}

\subsection{Experimental set-up for thermographic measurements}

In order to validate the calculations of the Atlas code, a thermographic system was developed. The main part of the 

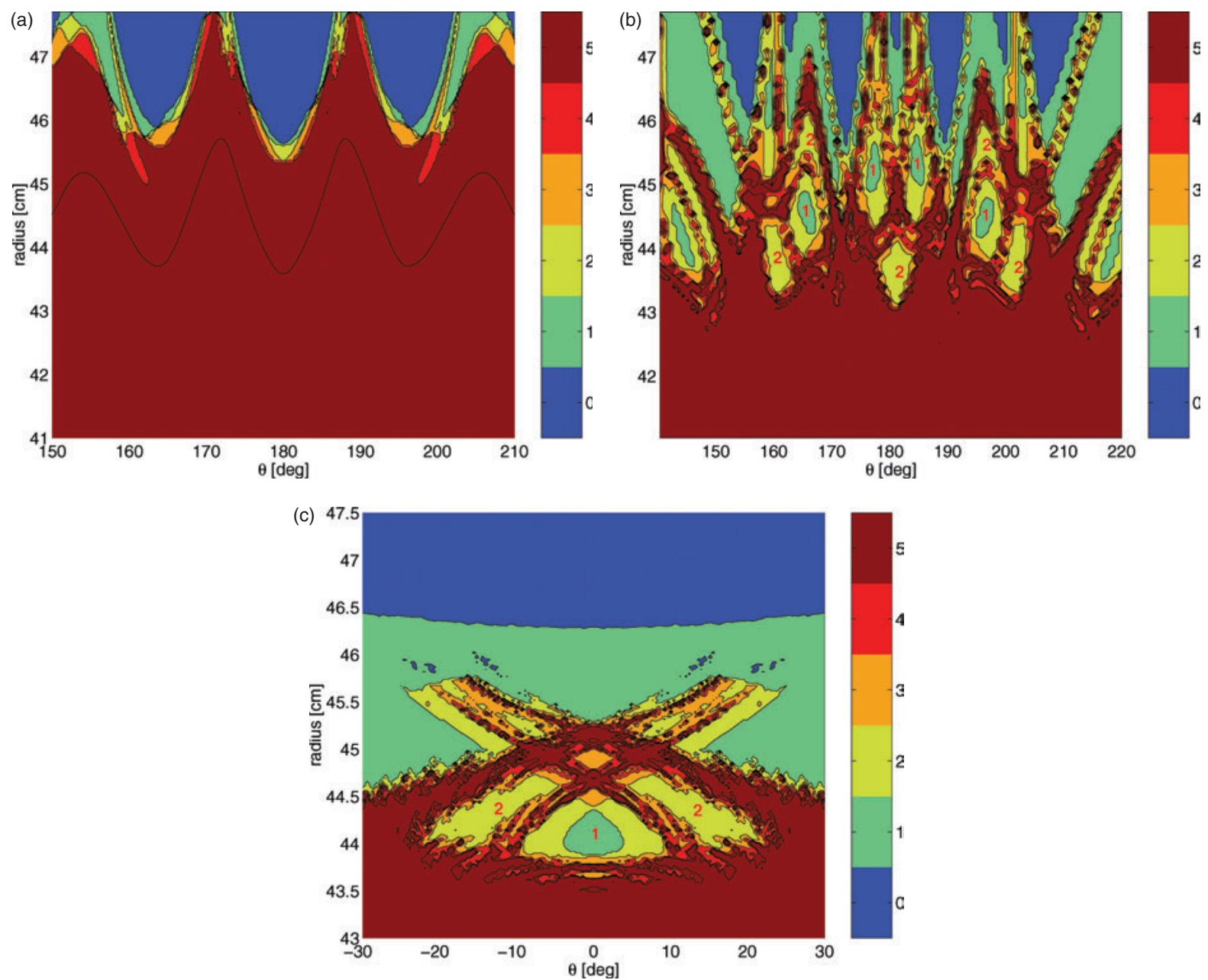

Figure 11. The laminar plots for two cases with dominant (a) ergodic region (plotted on the HFS; plasma current is $380 \mathrm{kA}$ ), $(b)$ and (c) laminar region (plotted on the HFS and LFS, respectively; plasma current is $500 \mathrm{kA})$. Other conditions are the same as in figure 8(c). The areas in $(b)$ and $(c)$ marked by the same number are interconnected.
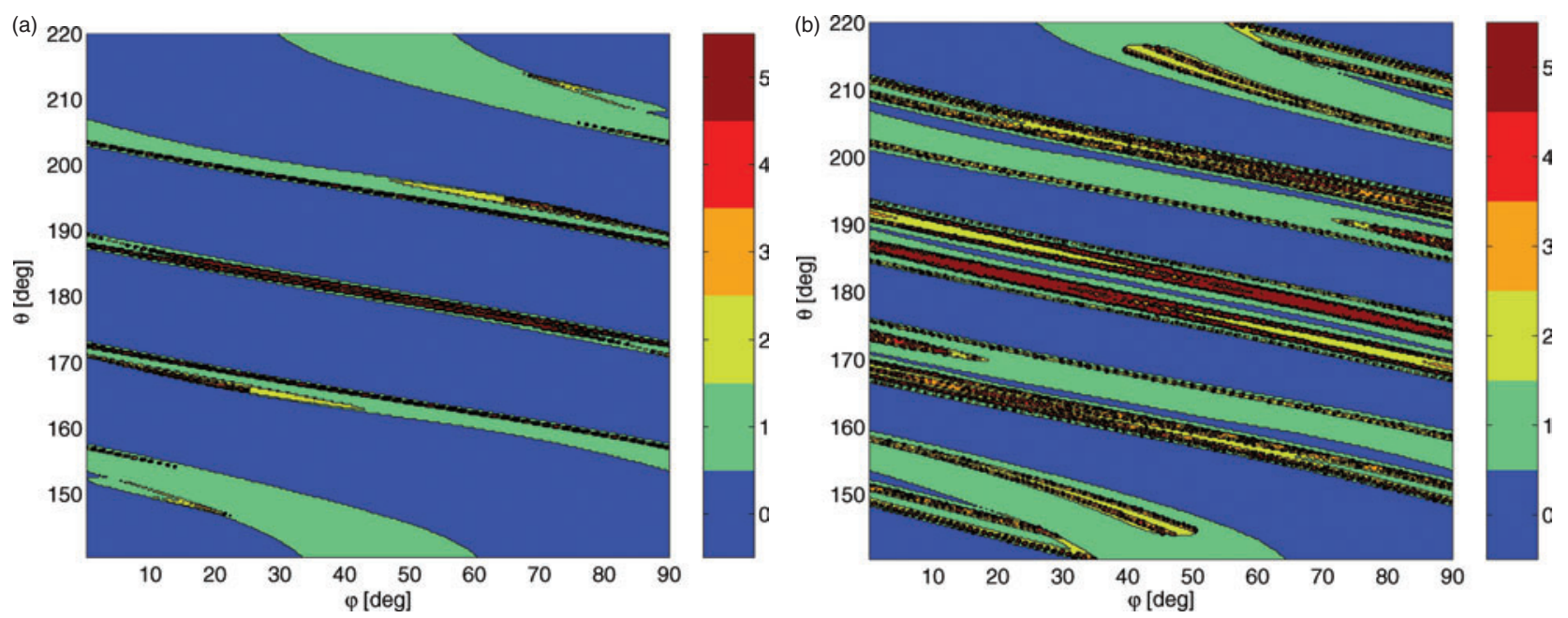

Figure 12. Contour plots of the magnetic footprints on the DED target plates for the same parameters as in figure 11.

set-up is a fast infrared camera, which detects radiation from a surface of the DED tiles. The active element of the camera is an InSb array focal plane, which consists of $320 \times 256$ pixels. The camera is sensitive to the spectral range $3-5 \mu \mathrm{m}$. The maximum time resolution of the camera is $2.5 \mathrm{~ms}(394 \mathrm{~Hz})$ with the whole array plane active. If one reduces the active size of the array to $128 \times 8$ pixels, the time resolution reaches $75 \mu \mathrm{s}$ $(13 \mathrm{kHz})$. The space resolution of the system is about $2 \mathrm{~mm}$. 
The measurements of heat fluxes for TEXTOR-DED operation
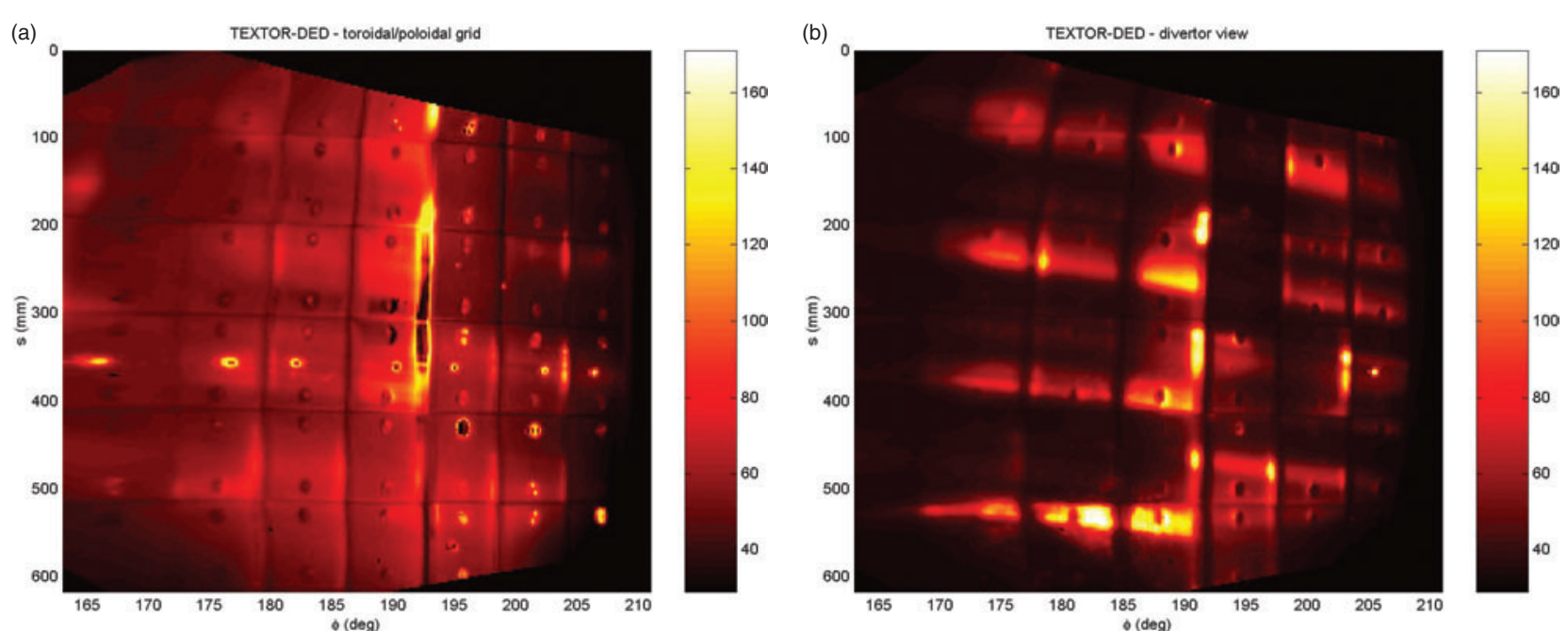

Figure 13. Temperature distribution pattern on the divertor target plates measured by the infrared camera. The colour scale corresponds to the temperature in ${ }^{\circ} \mathrm{C}$. (a) Discharge \#92596-lower ergodization, (b) discharge \#93100-higher ergodization.

The infrared pictures show that the alignment of the tiles is not perfect, which introduces difficulties for evaluating the data. For better visualisation of the stripes, the average over a few pictures just before the DED action was assumed as a background and removed from the pictures taken during the DED action. This enhancement was not used for the power flux evaluation. The pictures taken by the infrared camera were analysed using the LEOPOLD code [17], which was used to unfold the picture of the tiles and to prepare the data for heat flux calculations. An unfolded picture shows 5 rows of the tiles. The ordinate ( $s$ coordinate) represents the position along the poloidal angle measured in $\mathrm{mm}$; the abscissa is the toroidal angle. The toroidal range is about $50^{\circ}$, so that it does not correspond to the full period of the perturbation field $\left(90^{\circ}\right)$. The equatorial plane of the tokamak exists at about $s=300 \mathrm{~mm}$, between two rows of the tiles. The power fluxes were evaluated with the THEODOR code [18]. This is a two-dimensional code which takes into account the temperature dependent parameters.

\subsection{Results}

First experiments with the DED were performed with reduced DED and plasma currents due to technical problems. The level of ergodization was varied predominantly by shifting the plasma closer to or further away from the DED coils. The maximum value of the DED was $7 \mathrm{kA}$. Therefore, it was not possible to get a fully developed laminar region. However, the few experiments which were performed confirm the modelling. Two examples of measured temperature distributions are shown in figure 13. In both cases some parameters were similar: $I_{\mathrm{DED}}=7 \mathrm{kA}, B_{\varphi}=1.9 \mathrm{~T}$. The differences were in the plasma position, the poloidal beta and the plasma current. Shot \#92596 (figure 13(a)) was an Ohmic shot with $\beta_{\mathrm{pol}} \approx 0.25, I_{\mathrm{p}}=380 \mathrm{kA}$ and plasma position $R_{0}=1.74 \mathrm{~m}$. During discharge \#93100 (figure $14(b)$ ) a neutral beam was injected (about $0.5 \mathrm{MW}$ of the additional heating power, $\beta_{\mathrm{pol}} \approx 0.5, I_{\mathrm{p}}=400 \mathrm{kA}$ ) and the plasma position was $1.72 \mathrm{~m}$. The plasma during the second discharge was shifted towards the DED coils by $3 \mathrm{~cm}$ as compared to the first one. Such a shift increases significantly the level of ergodization. The change of the plasma current and poloidal beta also changes the level of the ergodization (as was discussed in section 4). The enhancement technique explained in the previous subsection was used for figure 13(a); therefore, the temperature is slightly overestimated and some darker spots are visible. The temperature pattern consists of four helical stripes. The width of the stripes is about $80 \mathrm{~mm}$. The 8 bright spots below the equatorial plane are produced by overheated Langmuir probes. The infrared picture of the DED tiles for discharge \#93100 shows that the power stripes are split. This means that the laminar layer is established. Due to misalignment of the tiles one can recognize that the particles hitting the wall in other halves of the stripe come from a different toroidal direction. This observation is consistent with the statement from section 4 that the pair of stripes is connected by the same flux tubes intersecting the wall. For a better illustration, a sketch is shown in figure 14 .

The structures obtained from the infrared measurements should be compared to the modelling with Atlas codes. Unfortunately, the applied model of the equilibrium field is valid only for the TEXTOR plasma if the plasma is shifted towards the DED tiles by less than 2 centimetres from the standard position $\left(R_{0}=1.75 \mathrm{~m}\right)$. The major radius of the plasma during shot \#93100 was at $1.72 \mathrm{~m}$ and the equilibrium model breaks down at this position. For discharge \#92596 the modelling could be done and it will be presented in the next subsection.

\subsection{Comparison between modelling and experimental results}

As mentioned, the applied model for the equilibrium field of TEXTOR is only valid for the plasmas with major radius within the range of $1.73-1.75 \mathrm{~m}$. Only a few discharges were performed with major radius in this range. The reason for that is that the maximal DED current was not reached, and to increase the effects of the DED, plasmas were shifted against the divertor target plates.

For discharge \#92596, the plasma was in the correct position for the equilibrium model applied in Atlas codes. 

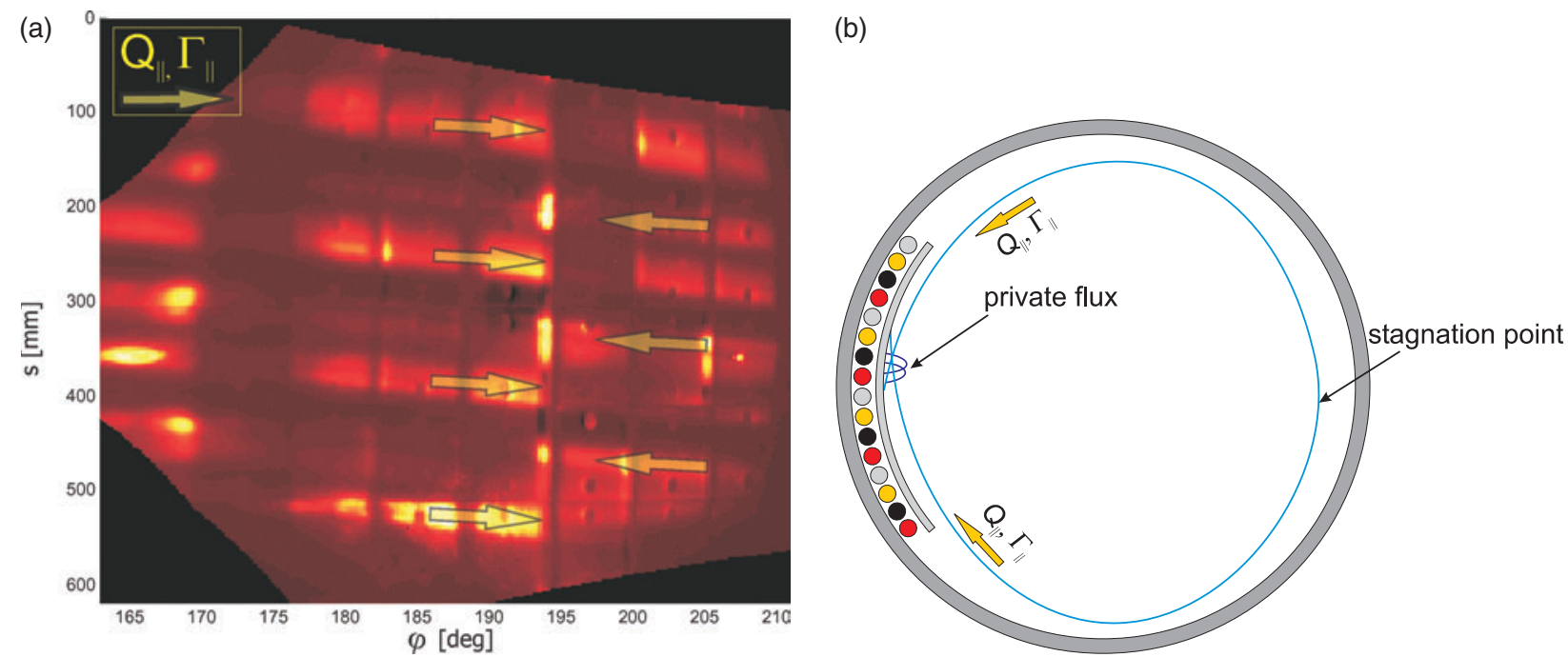

Figure 14. (a) Temperature distribution pattern on the divertor target plates for the same discharge as in figure 13(b), but with superimposed arrows indicating the direction of the incoming particles. (b) Sketch of the flux tube connecting two parts of the same power stripe. The DED coils are plotted on a left-hand side of the picture.

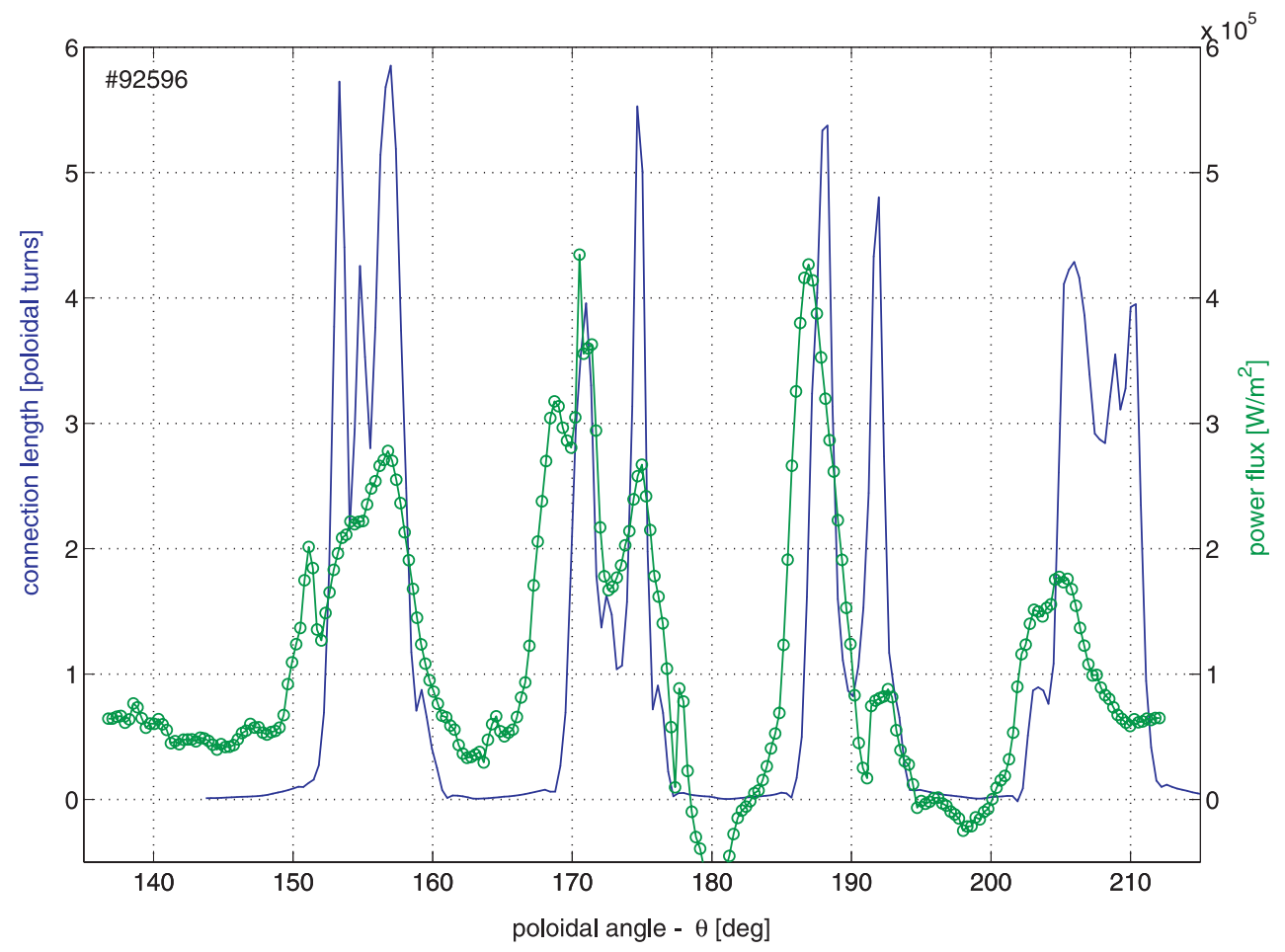

Figure 15. The heat flux (green curve, right ordinate) and the connection length (blue curve, left ordinate) profiles at toroidal angle $\varphi=187^{\circ}$ versus the poloidal angle.

To check if the topology of the magnetic field in the real plasma boundary corresponds to the model, the power flux profile was calculated along the tiles at the toroidal angle $\varphi=187^{\circ}$. To avoid incidental changes of the profile an average over the whole duration of the DED action was taken. For the same toroidal position the connection length profile along the DED tiles was calculated. The results are presented in figure 15. They show that most of the heat is brought from the plasma core to the wall by the field lines with relatively long connection lengths (more than 4 poloidal turns). This means that the ergodic region dominates in the plasma boundary.
The agreement between the modelling and the measurements allows us to study the topology of the magnetic field for shot \#92596 with the Atlas codes. The asymmetry in the values of the heat fluxes is caused mainly by the misalignment of the tiles. The results of the Atlas calculations are presented in figure 16. The width of the perturbed volume is about $6 \mathrm{~cm}$. For this shot the ergodic region still plays a significant role; however, there exists also the laminar zone (as seen in figure $16(b)$ ). The laminar zone consists predominantly of the one-poloidal-turn areas. The width of the footprints stripes is consistent with the observed power fluxes. 

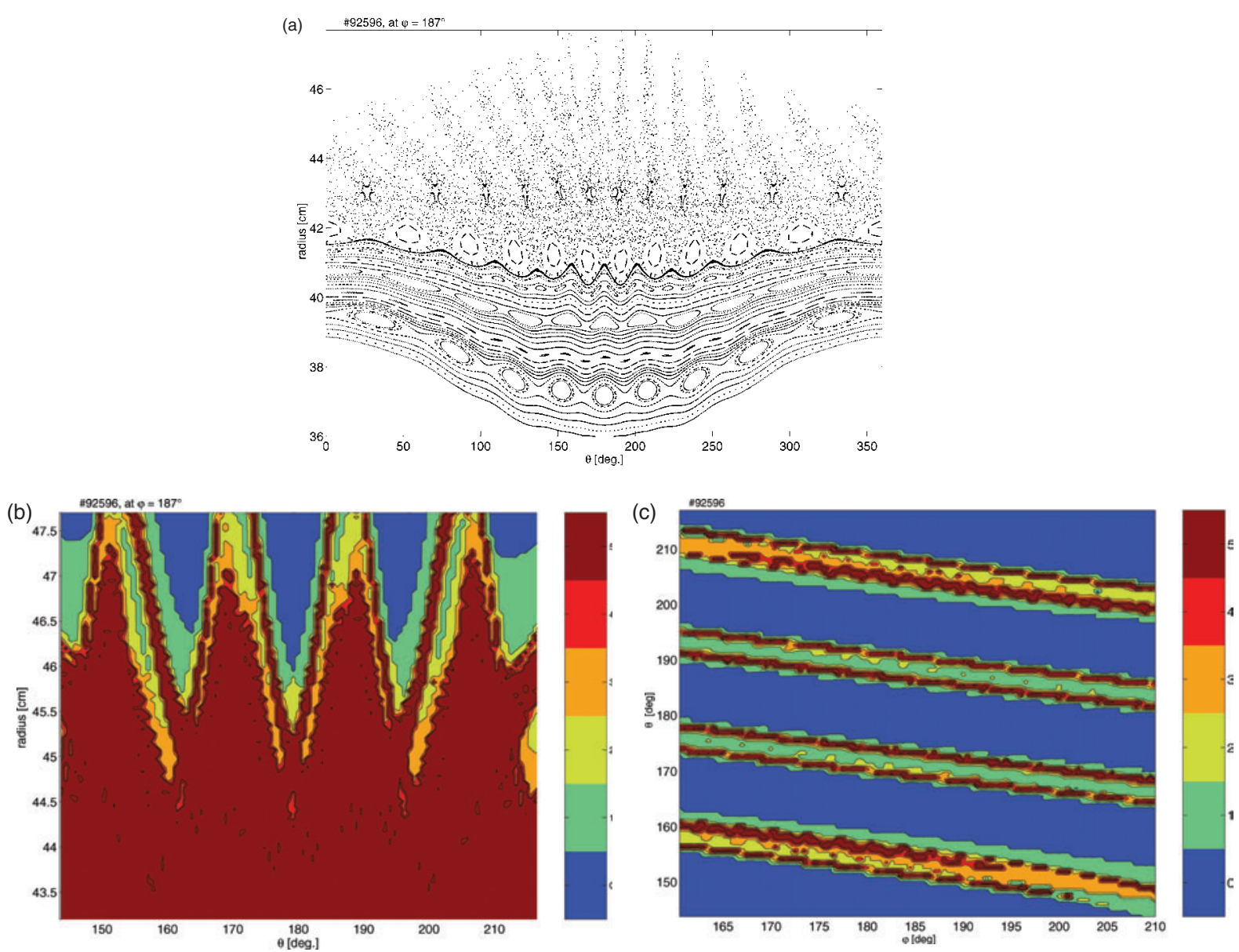

Figure 16. The results of the modelling with the Atlas codes of the magnetic field in the TEXTOR plasma edge for the discharge \#92596. $(a)$ Poincaré plot, $(b)$ laminar plot and $(c)$ footprints plot.

\section{Summary}

The action of the DED significantly modifies the structure of the magnetic field in the plasma boundary. The topological properties of the magnetic field in the TEXTOR-DED plasma boundary were considered. The codes which were developed are based on the fast, symplectic mapping scheme. The typical structure of the ergodized edge consists of two different layers: the ergodic region, where the field lines create a stochastic system, and the laminar region, where continuous flux tubes exist. The particle and heat transport are defined by both regimes. Depending on plasma parameters and the DED currents either the ergodic or laminar region dominate's. The characteristic magnetic footprint patterns are established on the divertor target plates. They are composed of four helical stripes, parallel to the coils. The particle and heat deposition pattern is defined by the structure of the magnetic footprints. The thermographic measurements confirm the model of the plasma boundary revealed with the Atlas codes; however, the equilibrium model applied in calculations needs further improvements.

\section{References}

[1] Federici G. et al 2001 Nucl. Fusion 41 1967-2137

[2] Ghendrih Ph., Grosman A. and Capes H. 1996 Plasma Phys. Control. Fusion 381653
[3] McCool S.C. et al 1989 Nucl. Fusion 29547

[4] Grosman A. et al 1998 Contrib. Plasma Phys. 3859

[5] Shoji T. et al 1992 J. Nucl. Mater. 196-198 296

[6] Takamura S. 1989 J. Nucl. Mater. 162-164 643

[7] Finken K.H. (ed) 1997 Fusion Eng. Des. 37 (special issue on Dynamic Ergodic Divertor)

[8] Finken K.H. et al 1999 J. Nucl. Mater. 266-269 495

[9] Abdullaev S.S., Finken K.H. and Eich Th. 2001 Phys. Plasmas 82739

[10] Abdullaev S.S., Finken K.H. and Spatschek K.H. 1999 Phys. Plasmas 6153

[11] Abdulaev S.S. et al 2003 Nucl. Fusion 43299

[12] Eich Th., Reiser D. and Finken K.H. 2000 Nucl. Fusion 40 1757

[13] Lichtenberg J.A. and Liebermann M.A. 1983 Regular and stochastic motion Applied Mathematical Science vol 38 (Berlin: Springer)

[14] Chirikov B.V. 1979 Phys. Rep. 52265

[15] Runov A.M., Reiter D., Kasilov S.V., Heyn M.F. and Kernbicher W. 2002 Phys. Plasmas 8

[16] Kobayashi M., Feng Y., Sardei F., Reiter D., Finken K.H. and Reiser D. 2003 3D numeric study on edge plasma transport in TEXTOR-DED Contributions at the 30th EPS on Controlled Fusion and Plasma Physics (Sankt Petersburg)

[17] Eich Th. 2003 Max Planck Institute, Garching, private communication

[18] Hermann A. et al 2001 Limitations for divertor heat flux calculations of fast events in tokamaks Contributions at the 28th EPS on Controlled Fusion and Plasma Physics (Madeira) 